\title{
Differentiation of trophoblast cells from human embryonic stem cells: to be or not to be?
}

\author{
R Michael Roberts ${ }^{1,3,4}$, Kyle M Loh7, Mitsuyoshi Amita ${ }^{1,3}$, Andreia S Bernardo ${ }^{8,9}$, \\ Katsuyuki Adachi ${ }^{2}$, Andrei P Alexenko ${ }^{1,3}$, Danny J Schust ${ }^{2}$, Laura C Schulz ${ }^{2}$, \\ Bhanu Prakash V L Telugu ${ }^{5,6}$, Toshihiko Ezashi ${ }^{1,3}$ and Roger A Pedersen ${ }^{9}$ \\ ${ }^{1}$ Division of Animal Sciences and ${ }^{2}$ Department of Obstetrics, Gynecology, and Women's Health, University of \\ Missouri, Columbia, Missouri 65211, USA, ${ }^{3} 240 b$ Bond Life Sciences Center, University of Missouri, \\ 1201 E. Rollins Street, Columbia, Missouri 65211-7310, USA, ${ }^{4}$ Department of Biochemistry, University of Missouri, \\ Columbia, Missouri 65211, USA, ${ }^{5}$ Animal Bioscience and Biotechnology Laboratory, Beltsville, Maryland 20705, \\ USA, ${ }^{6}$ Animal and Avian Sciences, University of Maryland, College Park, Maryland 20742, USA, ${ }^{7}$ Department of \\ Developmental Biology, The Stanford Institute for Stem Cell Biology and Regenerative Medicine, Stanford University \\ School of Medicine, Stanford, California 94305, USA, ${ }^{8}$ National Institute for Medical Research, Medical Research \\ Council, Mill Hill, London, UK and ${ }^{9}$ The Anne McLaren Laboratory for Regenerative Medicine, Stem Cell Institute, \\ University of Cambridge, West Forvie Building, Robinson Way, Cambridge CB2 OSZ, UK
}

Correspondence should be addressed to R M Roberts; Email: robertsrm@missouri.edu or to R A Pedersen; Email: Roger@stemcells.cam.ac.uk

\begin{abstract}
It is imperative to unveil the full range of differentiated cell types into which human pluripotent stem cells (hPSCs) can develop. The need is twofold: it will delimit the therapeutic utility of these stem cells and is necessary to place their position accurately in the developmental hierarchy of lineage potential. Accumulated evidence suggested that hPSC could develop in vitro into an extraembryonic lineage (trophoblast (TB)) that is typically inaccessible to pluripotent embryonic cells during embryogenesis. However, whether these differentiated cells are truly authentic TB has been challenged. In this debate, we present a case for and a case against TB differentiation from hPSCs. By analogy to other differentiation systems, our debate is broadly applicable, as it articulates higher and more challenging standards for judging whether a given cell type has been genuinely produced from hPSC differentiation.
\end{abstract}

Reproduction (2014) 147 D1-D12

\section{Introduction}

Case for differentiation of human embryonic stem cells into trophoblast cells (proposers: $R$ M Roberts, $M$ Amita, K Adachi, A P Alexenko, D J Schust, L C Schulz, B P V L Telugu, and T Ezashi).

Ethical and regulatory concerns limit our ability to move from animal models to humans in an effort to follow trophoblast (TB) development from the blastocyst to the fully functional placenta (Enders 2000, Carter 2007). As a result, limited morphological information is available on initial stages of human TB invasion and placentation (Enders 2000). What is generally inferred is that cytotrophoblast (CTB) cells derived from blastocyst trophectoderm differentiate into either villous CTB or extravillous CTB. The former provides the multilayered structures that ultimately form the chorionic villi and syncytio TB (Georgiades et al. 2002, Malassine \& Cronier 2002), a cell layer that makes direct contact with maternal blood and is characterized by the production of human chorionic gonadotropin (hCG) and other placental hormones. Extravillous CTB, by contrast, assumes an HLA-G+ phenotype, and a subpopulation modifies maternal spiral arteries to increase blood flow through the placental bed.

In absence of a ready source of trophoblast stem cells (TSCs), the best in vitro models to study the development of these TB lineages have, until recently, been choriocarcinoma cells, such as JAr and JEG3 lines, immortalized extravillous CTB cell lines, and primary CTB cultures from placenta (Ringler \& Strauss 1990, Schulz et al. 2008). However, there are major shortcomings to each of these models. Importantly, none of them allows cell lineages to be followed from their stem 
cell progenitors to fully differentiated syncytioTB and extravillous TB cell types (Schulz et al. 2008, Genbacev et al. 2013).

An experimental system in which human embryonic stem cells (hESC) are driven toward TB in response to bone morphogenetic protein 4 (BMP4) was first described by Thomson's group (Xu et al. 2002, Xu 2006) and later by others (Besser 2004, Gerami-Naini et al. 2004, Golos et al. 2006, Chen et al. 2008, Wu et al. 2008, Zhang et al. 2008, Douglas et al. 2009, Erb et al. 2011, Marchand et al. 2011, Sudheer et al. 2012) including ourselves (Das et al. 2007, Schulz et al. 2008, Gupta et al. 2012, Amita et al. 2013, Telugu et al. 2013). This phenomenon has generally been observed with the FGF2-dependent 'primed' type of ESC, such as those derived from the inner cell mass of human (Xu et al. 2002) and rabbit (Tan et al. 2011) blastocysts, and generally not in naïve type ESC as represented, for example, by the LIF/STAT3-dependent mouse ESC (mESC). However, there is at least one exception where BMP4 exposure generated TB from naïve type cells, namely when mESC were cultured on a laminin substratum (Hayashi et al. 2010).

This BMP4-dependent model system has allowed the very early stages of TB lineage specification and expansion from pluripotent progenitors to be examined over time and subsequent differentiation of these early progenitor cells to be followed along specific sublineages. Morphologic differentiation in response to BMP4 is readily visible as the spread of larger, flattened cells proceeding from the periphery inwards ( $\mathrm{Xu}$ et al. 2002, Das et al. 2007, Amita et al. 2013). Microarray analysis performed over time following exposure to BMP4 revealed immediate upregulation of transcription factors associated with TB emergence, with little evidence for major upregulation of lineage markers for mesoderm, endoderm, or ectoderm (GEO GSE10469; Marchand et al. 2011, Ezashi et al. 2012, Sudheer et al. 2012). There was a gradual loss of expression of 'stemness genes', increased expression of genes associated with differentiated TB, and emergence of morphologically recognizable syncytium-like cells, which was accompanied by release of placental hormones (Das et al. 2007, Sudheer et al. 2012, Amita et al. 2013). When the ability of cells to migrate through Matrigel was assessed, cultures maintained under conditions designed to minimize differentiation, i.e. with FGF2 present and BMP4 absent, showed little or no invasiveness (Amita et al. 2013, Telugu et al. 2013), but significant migration was observed in cultures treated with either BMP4 (Telugu et al. 2013) or BMP4 plus the Activin A signaling inhibitor A-83-01 and FGF2 signaling inhibitor PD173074) (BMP4/AP treatment) (Amita et al. 2013). By day 5 of treatment, invasive potential had been raised $\sim 160$-fold by BMP4/AP and 65 -fold by BMP4 alone and the cells that had migrated to the underside of the invasion chamber membrane were all positive for KRT7, while a majority were positive for HLA-G (Amita et al. 2013, Telugu et al. 2013).

The original medium used to observe BMP4-driven differentiation consisted of $80 \%$ DMEM/F12, $20 \%$ knockout serum replacement (KOSR; Invitrogen), and FGF2 (4 ng/ml) (Amit et al. 2000, Xu et al. 2002). It was defined, but for the fact that the cells were grown on either a 'feeder' layer of irradiated mouse embryonic fibroblasts (MEF) or on MEF-conditioned medium. Initially, BMP4 was added to this medium in the continued presence of FGF2 (Xu et al. 2002, Das et al. 2007), but it was quickly realized that removing FGF2 enhanced differentiation to TB (Das et al. 2007, Marchand et al. 2011, Sudheer et al. 2012). Indeed, in presence of high FGF2 concentrations, BMP4 induced expression of genes characteristic of mesoderm (Vallier et al. 2009a, Yu et al. 2011). BMP4-directed TB differentiation was further improved by inclusion of the Activin inhibitor SB431542 (Wu et al. 2008, Erb et al. 2011). Activin A promotes transcription of key pluripotency genes (Xu et al. 2008, Vallier et al. 2009b), and its own signaling probably competes with that of BMP4 for SMAD intermediates. Recently, the Roberts's lab has employed the chemically defined mTeSR 1 medium supplemented with FGF2 $(100 \mathrm{ng} / \mathrm{ml})$ and TGF $\beta$ $(0.6 \mathrm{ng} / \mathrm{ml})$ to grow hESC (Amita et al. 2013), while differentiation was induced, as before, with $10 \mathrm{ng} / \mathrm{ml}$ BMP4 in DMEM/F12/KOSR medium, with or without MEF conditioning and in the absence of FGF2. As it turns out, MEF conditioning is unnecessary to drive TB differentiation (Amita et al. 2013), so that it is now possible to conduct both the hESC growth phase and differentiation phase under chemically defined conditions. Addition of inhibitors of Activin (A-83-01) and FGF2 signaling (PD173074) further accelerates TB differentiation (Amita et al. 2013).

Despite the compelling evidence supporting the BMP4/hESC model, Bernardo et al. (2011) concluded: 'we have no evidence to support the thesis that hESCs have the capacity to generate TB simply by addition of BMP to their growth medium in the presence or the absence of FGF2 or with Activin/Nodal inhibition' and that 'BMP induces human pluripotent stem cell (hPSC) and mouse PSC primarily to form mesoderm'. In that study, the hESCs were cultured on a chemically defined, serum-free, F12-based medium, supplemented with $10 \mathrm{ng} / \mathrm{ml}$ recombinant Activin A and $12 \mathrm{ng} / \mathrm{ml}$ recombinant FGF2, on a gelatin-coated substratum soaked in FBS. During differentiation, the BSA in this medium was replaced with polyvinyl alcohol, and the content of FGF2, Activin A, BMP4, and various inhibitors adjusted according to the differentiation protocol employed. The fact that Bernardo et al. chose to use this medium rather the one used by earlier investigators to draw inferences about the BMP4/hESC model clearly complicates the present debate on the validity of the BMP4/hESC model. 
The results from the Roberts's lab that differed most significantly from those of Bernardo et al. (2011) can be summarized as follows:

i) They showed that three genes associated with embryonic and extra-embryonic mesoderm, namely $K D R$ (FLK1), VCAM1, and TBX4, were quickly upregulated by BMP4 (Ezashi et al. 2012, Amita et al. 2013). Our data, however, indicated that, with the exception of $K D R$, which is expressed in human extravillous CTB (lacob et al. 2008), these genes are expressed relatively weakly in hESC, with very little upregulation by BMP4 over the initial 3 days of exposure.

ii) Bernardo et al. (2011) reported that only $4-8 \%$ of BMP-treated cells expressed KRT7 at day 7, whereas the Roberts's lab showed that virtually all cells in the colonies of BMP-treated cells became positive for KRT7 by day 2 (BMP4/AP) or day 4 (BMP4). Even when the Roberts's lab used the chemically defined medium (CDM) medium of Bernardo et al., BMP4 converted over $40 \%$ and BMP4/AP over $70 \%$ of the cells to a KRT7 + state within 5 days (Amita et al. 2013).

iii) Bernardo et al. (2011) noted only 'sporadic' multinucleated cells and suggested that these were not syncytio TB but some mesoderm derivative. In the BMP4/hESC model, large areas of the colonies form syncytia, and these multinucleated cells are strongly positive for syncytio TB markers.

iv) In the hands of the Roberts's lab, WA01 and WA09 produced large quantities hCG by day 9 in response to BMP4 with or without A-83-01 and $\mathrm{PD}$, but when the same cells were treated with BMP4/AP on the CDM medium and substratum, described by Bernardo et al., in the absence of the pluripotency factors, FGF2, and Activin A, the cells produced only about $1 \%$ of that amount of hormone.

v) According to Bernardo et al. (2011), BMP4-treated hESC did not express HLA-G, while in the Roberts's lab strong signals for both surface and internal HLA-G were observed (Das et al. 2007, Amita et al. 2013).

vi) The Roberts's lab observed no upregulation in $T$ (Brachyury; marker of early-stage mesoderm and endoderm) under BMP4/AP conditions and only weak expression with BMP4 alone and then only under high $\mathrm{O}_{2}$ conditions (Amita et al. 2013).

What then can explain the discrepancies between our two papers? The nature of the cells is not an issue. In our hands, for example, WA09 cells provide qualitatively similar outcomes to WA01 cells (Amita et al. 2013). Possibly, it is the nature of the culture medium and substratum that influences the directionality of differentiation. For example, does either the continued presence of Activin A in the CDM medium or the soaking of the matrix in FBS inhibit TB formation and bias differentiation in favor of mesoderm even when a potent Activin A inhibitor is present? The Roberts's lab does not quarrel with the notion that FGF2 and Activin A in combination with BMP4 can promote both endoderm and mesoderm in proportions that depend on the relative concentrations of the three growth factors (Zhang et al. 2008, Vallier et al. 2009a, Yu et al. 2011). However, it is unambiguously clear that BMP4 in the absence of FGF2 and Activin A signaling induces hESC to form TB. Our concern is that there may be a broad perception that the BMP4/hESC model for TB differentiation is somehow flawed and that this slant might be reflected in the manner in which manuscripts and grant proposals that incorporate the model are reviewed.

\section{Case against differentiation of hESC into TB cells (opposers: K M Loh, A S Bernardo, and R A Pedersen)}

The developmental competence of cells - what repertoire of committed fates a particular progenitor can access - is pertinent to both developmental and stem cell biology. For example, the mouse embryo's pluripotent epiblast cells, which can form all cell types of the body proper, have lost the ability to form any extraembryonic lineage, such as TB or hypoblast (Gardner \& Rossant 1979). Unexpectedly, hESCs, which are derived from and are thought to correspond to pluripotent epiblast, have been reported to harbor TB potential (Xu et al. 2002), a view supported by other studies (Erb et al. 2011, Drukker et al. 2012, Sudheer et al. 2012, Amita et al. 2013). The unanticipated TB competence of hESC has led to suggestions that hESC are either 'totipotent' in multilineage potential or altered in vitro derivatives of epiblast with aberrantly expanded lineage potential (Silva \& Smith 2008).

Studies reporting the derivation of TB from hESCs in vitro attribute this outcome to BMP signaling ( $\mathrm{Xu}$ et al. 2002, Erb et al. 2011, Drukker et al. 2012, Sudheer et al. 2012, Amita et al. 2013). Such a role of BMP was surprising, as genetic perturbations in mouse $\left(B m p r 1 a^{-1-}\right.$ and $B m p r 2^{-1-}$ mutants) have not revealed any defect in TB development in vivo (Mishina et al. 1995, Beppu et al. 2000). Rather such in vivo genetic studies of Bmpr1a ${ }^{-1-}$ and $B m p r 2^{-1-}$ mutants demonstrate a critical role for BMP signaling in mesoderm development, a conclusion recently corroborated in vitro using both hESC differentiation and mouse epiblast explant approaches (Bernardo et al. 2011, Loh et al. 2014).

Examining BMP signaling from an embryonic perspective could provide insights into its possible role in TB development. To the best of our knowledge, there is no evidence for robust BMP signaling in pre-implantation trophectoderm, as would be evidenced by expression of the appropriate receptors. The extraembryonic ectoderm 
of post-implantation mouse embryos actively transcribes Bmp4 and Bmp8b; however, significant amounts of pSmad1/5/8 signaling have not been observed there (Di-Gregorio et al. 2007).

Why then would BMP be expected to induce hESCs to differentiate into TB? In retrospect, from a developmental perspective, BMP-induced differentiation of hESCs into TB would not have been anticipated. Nevertheless, a number of studies report BMP-based techniques to apparently generate TB from hESCs, most prominently the application of BMP signaling in conjunction with inhibitors of TGF $\beta$ and FGFR/MAPK signaling (Erb et al. 2011, Sudheer et al. 2012, Amita et al. 2013). Presumably, combined TGF $\beta$ and FGFR/MAPK inhibition blocks the expression of pluripotency factors and primitive streak genes, thus diverting hESC from other mutually exclusive prospective fates to help consolidate putative TB commitment. In these various culture conditions, cells have emerged that express i) TB-associated genes (e.g. CDX2 and HAND1) and keratins, ii) secrete placental hormones (e.g. hCG), and/or iii) are multinucleated ( $\mathrm{Xu}$ et al. 2002, Erb et al. 2011, Drukker et al. 2012, Sudheer et al. 2012, Amita et al. 2013).

At the crux of this matter is the question of how markers can be used to identify with confidence the developmental lineages that arise during hESC differentiation in vitro. Precisely defining cell identity by marker analysis alone can be confounded by three factors: i) TB 'marker genes' may be expressed in other embryoderived lineages (e.g. mesoderm) besides TB, thereby confounding the diagnostic utility of these markers; ii) a large number of potential lineage fates must be examined and excluded in order to rule out a developmentally 'mixed' phenotype and thereby identify cells as bona fide TB, and iii) bona fide TB cells should manifest a substantial set of additional expected characteristics. These issues lead us to identify eight decisive areas that could be addressed in future work to ascertain whether hESC truly harbor TB competence, thus forming a framework for future progress and dialogue.

i) It has been recently appreciated that $C D X 2$, HAND1, and GATA3 - typically viewed as salient hallmarks of TB - are also expressed in posterior mesoderm, warranting caution in use of these in assigning TB identity to cells formed during BMPinduced differentiation of hESCs (Bernardo et al. 2011). This caveat applies equally to differentiation of mESCs induced by BMP treatment (Hayashi et al. 2010). Keratin induction by TB differentiation protocols has been cited as key evidence for TB identity (Amita et al. 2013), yet keratins have broad embryonic expression in a variety of lineages. Similar issues apply to using an epithelial-like morphology of these differentiated cells to definitively assign TB identity (Erb et al. 2011, Amita et al. 2013). ii) Another issue is that candidate hESC-derived TB does not detectably express SOX2 and EOMES (Sudheer et al. 2012, Amita et al. 2013), which are highly expressed in the mouse extraembryonic ectoderm in vivo and TSCs in vitro, and are essential for TSC specification ( Russ et al. 2000, Avilion et al. 2003, Keramari et al. 2010). The absence of SOX2 and EOMES during in vitro differentiation of hESCs toward putative TB raises the question of whether they actually acquire a bona fide TB identity.

iii) Placental hormone secretion following hESC differentiation is compelling, but it is unclear that this precisely identifies the TB lineage, since the placental expression domains of these hormones have not been specifically mapped in vivo. Thus, it remains formally possible that these hormones could be expressed by the extraembryonic mesoderm component of the chorioallantoic placenta as well as by TB. Indeed, there is evidence to suggest that such BMP-induced hESC populations actually correspond to extraembryonic mesoderm (Bernardo et al. 2011).

iv) Though cardinal TSC markers SOX2 and EOMES are not expressed, a variety of other TB genes (e.g. ELF5 and AP2 family members) are transcriptionally upregulated to some extent during hESC differentiation (Xu et al. 2002, Amita et al. 2013). However, these genes are minimally expressed in undifferentiated hESCs, which may exaggerate their fold-change of upregulation, and they may not actually be detectable at the level of protein.

v) BMP-containing culture conditions have also induced the formation of multinucleated cells, which are thought to indicate fused syncytiotrophoblast (e.g. Drukker et al. (2012)). Nevertheless, it has been shown that even undifferentiated ESCs can readily fuse with heterologous cell types to form multinucleated syncytia (Ying et al. 2002).

vi) HLA-G expression by putative hESC-derived TB has been proclaimed as one measure of TB identity (Amita et al. 2013), as HLA-G is expressed by human extravillous TB in vivo (Bernardo et al. (2011) and references therein). Therefore, it would be a good gauge of TB identity. However, other attempts have not detected HLA-G expression, even using sensitive flow cytometry methods (Bernardo et al. 2011, Sudheer et al. 2012), regardless of the presence or absence of KOSR in the medium (Supplementary Fig. 4 of Bernardo et al. 2011). A complementary approach to assess the identity of these cells would be to characterize their expression of HLAA/HLA-B, as these HLAs are expressed in fetal lineages such as mesoderm but not in extravillous TB (Bernardo et al. (2011) and references therein). 
However, putative hESC-derived TB, differentiated in either CDM or KOSR, continuously express HLA-A and/or HLA-B (Bernardo et al. 2011). This remains to be tested by others.

vii) If differentiating hESCs have decisively entered the TB lineage, this should be reflected at the levels of both gene expression and chromatin. However, the promoter of ELF5 (a definitive TB hallmark) remains largely methylated in hESCdifferentiated TB populations (Bernardo et al. 2011), raising the question of whether observed ELF5 mRNA induction upon hESC differentiation (Amita et al. 2013) reflects leaky expression instead of robust upregulation. Although it remains possible that a small fraction of cells that emerge from these culture conditions are bona fide TB with complete ELF5 demethylation, such cells were not abundant enough to be captured by bisulfite sequencing. In any case, comprehensive chromatin state mapping of putative hESC-derived TB (Xie et al. 2013) should enable chromatin-level assessment of ELF5 and other hallmark TB genes, and thereby determine whether their chromatin state resembles that of placenta.

viii) To fully address whether hESC have TB competence, systematic protocols to elicit putative TB from hESC must be more widely adopted so that these cells can be analyzed by different groups. The use of complex culture systems containing, for example, mouse feeder cells or feederconditioned medium (Erb et al. 2011, Amita et al. 2013), or animal serum (Drukker et al. 2012) or even KSR (Xu et al. 2002, Amita et al. 2013) complicates reproducibility by other groups. Moreover, neither feeder cells nor animal serum represent a physiologic context for TB development, as these contain undefined factors that may influence differentiation in various ways. Ultimately, the adoption of CDM for differentiation in the complete absence of serum or feeders (Bernardo et al. 2011) would be ideal to ensure that differentiation protocols can be consistently evaluated throughout different laboratories. Such CDM offer highly controllable culture environs to precisely define the effects of developmental signaling-perturbations (e.g. BMP treatment) on hESC differentiation without being confounded by undefined factors (Bernardo et al. 2011, Loh et al. 2014).

Overall, these issues emphasize the need to precisely map molecular markers throughout multiple stages of human TB development in vivo (Niakan et al. 2012) and to establish TSC lines in vitro from human embryos. Accurately defining the molecular portrait of bona fide human TB is obligatory to fully evaluate whether putative hESC-derived TB is akin to its in vivo counterpart. This is especially important because significant cis-regulatory evolution between the human and mouse lineages has markedly distinguished TB development in these two species (e.g. Niakan et al. (2012)), therefore hESC differentiation efforts must reciprocally be guided by the knowledge of the human developmental process.

Altogether, it remains possible that hESCs harbor TB potential, but that the necessary extrinsic signaling conditions to expose such competence have so far remained elusive. Alternatively, presumptive epigenetic perturbations could resuscitate dormant TB potential, as DNA methyltransferase disruption reportedly enhances mESC differentiation toward TB (Ng et al. 2008), which is a developmentally inaccessible fate for mESCs. Indeed, from a developmental perspective, the peri-implantation epiblast in vivo (Gardner \& Rossant 1979) and mESCs in vitro (Silva \& Smith 2008) are generally refractory to TB differentiation. Because hESCs are thought to reflect an even later stage of development (reminiscent of the post-implantation epiblast), it is difficult to reconcile the known sequence of lineage commitments during early mammalian embryogenesis with an apparent TB competence of hESC. Nevertheless, such theoretic considerations should not deter experimental work to more fully explore whether hESC can indeed have close encounters of the TB kind.

\section{Debate}

Response to case against case for differentiation of hESC into TB cells (proposers: $R$ M Roberts, M Amita, K Adachi, A P Alexenko, D J Schust, L C Schulz, $B P V L$ Telugu, and T Ezashi)

The Pedersen laboratory raises several issues that they believe to argue against the TB-like properties of the cells generated by BMP4 treatment of hESC (points 1, 3, 5, and 6) and suggest that additional criteria (points 2, 4, and 6) need to be met before TB status can be accepted. What Pedersen's arguments lack is any evidence that the cells are not $\mathrm{TB}$, other than the preconceived notion, based largely on mouse data, that epiblast-derived cells should not be able to embark on this differentiation pathway.

Just as 'one swallow does not a summer make', one marker gene product does not TB make. Certainly, the expression of any one marker or even several together would not constitute proof that the BMP-hESC had converted to TB, but the combined weight of evidence from numerous studies employing a wide range of earlyand late-gene markers, including hundreds from microarray analyses, implies that these cells are TB (Fig. 1 and GEO GSE10469). For example, recent genome-wide expression profiling shows that BMP-hESC cluster with early-gestation placenta and away from all other fetal 


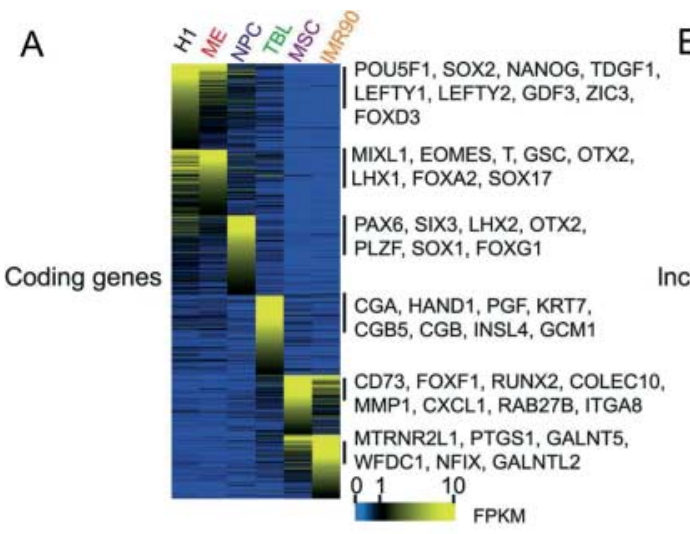

C

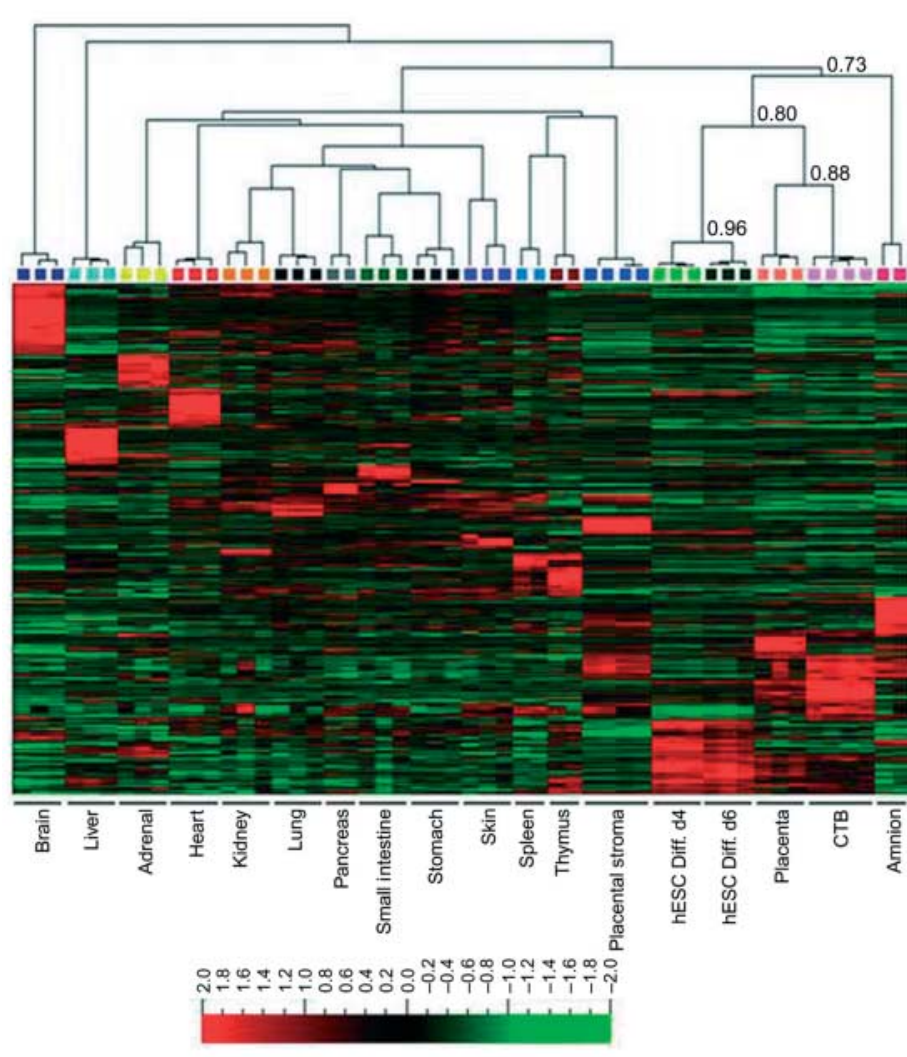

B

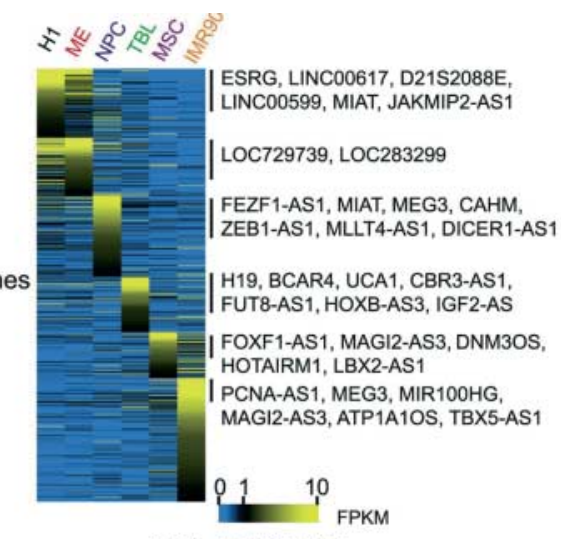

BMP5 BMP10 BMP13 $\begin{array}{ccc}100 & 100 & 1 \\ \mathrm{ng} / \mathrm{ml} & \mathrm{ng} / \mathrm{ml} \\ \mu \mathrm{gg} / \mathrm{ml}\end{array}$

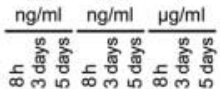

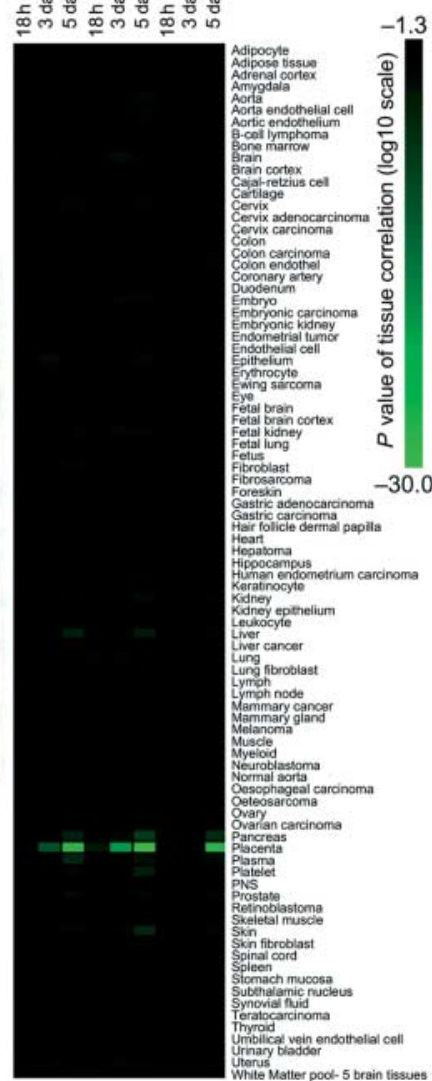

Figure 1 Proposer's view: examples of TB lineage restricted transcripts present in hESC treated with bone morphogenetic proteins (BMPs). The data were derived from three independent laboratories by different microarray methods. The top panel (Xie et al. 2013) compared heat maps for transcripts of coding genes (A) and long non-coding RNA (B) from $\mathrm{H} 1$ cells (lane 1 ) and $\mathrm{H} 1$ cells differentiated along various lineages by standardized protocols (ME, mesoendoderm; NPC, neural progenitor cells; TBL, trophoblast-like cells; MSC, mesenchymal stem cells; IMR90, primary human fetal lung fibroblast cells). Differentiation to TBL was achieved with $50 \mathrm{ng} / \mathrm{ml} \mathrm{BMP4}$ in FGF2-minus medium for 5 days (TBL). The study demonstrated that known lineage markers were strongly associated with the anticipated lineages, and that the trophoblast-like cells were distinct from the other lineages, including mesoendoderm, where differentiation had been driven with FGF2 and BMP4 in combination for $48 \mathrm{~h}$. (C), Li et al. (2013) compared expression profiles of $\mathrm{H} 9 \mathrm{hESC}$ that had been treated with $10 \mathrm{ng} / \mathrm{ml} \mathrm{BMP4}$ in FGF2-minus feeder-conditioned medium for either 4 or 6 days (hESC Diff.d4 and hESC Diff.d6 respectively) with whole, first-trimester placenta (Placenta), isolated cytotrophoblast (CTB) from such placentas, and a number of other tissues. Note that the differentiated hESC at both time points clustered most closely with placenta and CTB and were distinct from placental stroma, amnion, and other representative tissues on the array. (D) Lichtner et al. (2013) showed that, in addition to BMP4, BMP5, BMP10, and BMP13 in the absence of FGF2 drove hESC to the TB lineage. The transcriptional signatures of $\mathrm{H} 1 \mathrm{hESC}$ treated with the various BMP sub-types for $18 \mathrm{~h}, 3$, and 5 days were compared with those of various tissues and anatomical compartments. The analysis identified placenta as the tissue that most resembled the BMP-treated hESC $(P<0.05)$. 
tissues, including mesoderm-derived amnion and placental stroma (Li et al. 2013). That study also confirms with a different antibody that HLA-G is expressed (point 6 of Loh et al.). Another recent paper has shown that BMP-hESC resemble placental TB not just in the expression of coding genes but also in the composition of long non-coding RNA species and in 'epigenetic status' (Xie et al. 2013; Fig. 1A and B). Figure 1D also illustrates that the gene expression profiles of $\mathrm{H} 1 \mathrm{hESC}$ exposed to different members of the BMP family for increasing periods of time correlated most closely with that of the placenta and not with a broad and representative selection of other organs (Lichtner et al. 2013). Finally, the arguments against are mistaken in that BMP-hESC do not express SOX2 and EOMES (point 2). They do so robustly for the initial $24 \mathrm{~h}$ after BMP4 exposure and only later does expression decline (GEO GSE10469). Moreover, with the exception of placental hormone transcripts, the qPCR data of Amita et al. (2013), and all the array data of Telugu et al. (2013), were presented as normalized values and not as fold-change (see point 4 of Case against differentiation of hESC into TB cells).

This returns us to the issue as to why the results of (Bernardo et al. 2011) differ so dramatically from those of us who have employed the BMP-hESC model. The case against comments that the use of MEF-conditioned medium (Xu et al. 2002, Amita et al. 2013) is a confounding feature of the differentiation protocol. However, the generation of TB from hESC can be readily performed in a CDM in the absence of products of MEF (Amita et al. 2013; Fig. S6B). Moreover, the Roberts's lab have argued that the medium used by Bernardo et al. (2011) is not only sub-optimal for culturing hESC, but far from appropriate for testing the soundness of the BMPhESC model (Amita et al. 2013). In essence, the line of reasoning of Pedersen's lab boils down to the idea that TB emergence from hPSCs with the features of epiblast simply should not happen, a premise based entirely on what is perceived to occur during mouse development. Given the differences in early embryonic development between the species, is it not likely that this BMP-directed differentiation to TB may not be homologous to the signaling that generates trophectoderm and its immediate lineage descendants in mouse but, instead, reflects a different stem cell niche found in the early human placenta?

In summary, the counter arguments of Pedersen et al. fail to weaken our confidence in the validity of the BMPhESC model of TR. This controversy could have been averted if Bernardo et al. (2011) had reproduced the culture conditions used in the prior studies that had employed the model to study TB development, and by describing their own culture procedures in greater detail such that they could be readily reproduced by others.

Note Added in Proof: two recent reports have shown that two kinds of mouse PSC generated in hitherto unconventional ways, namely by in vivo reprograming (Abad et al. 2013) and 'stimulus-triggered acquisition of pluripotency' (STAP) (Obokata et al. 2014), respectively, can contribute to both the placenta and embryo proper after injection into blastocysts. The former can also give rise to regions of TB-like cells in teratomas, while the STAP cells differentiate along the TB lineage in response to FGF4, the standard growth factor used to grow mouse TSCs. Although it is not clear whether the epigenetic states of these cells are similar and how they acquired totipotent characteristics during their derivation, it is apparent that the dogma that says pluripotent cells derived from anything, but a totipotent blastomere cannot become TB should be rejected.

\section{Response to case for differentiation of hESC into TB cells (opposer: K M Loh, A S Bernardo, and R A Pedersen)}

To consider TB induction from hESCs as a valid model of human TB development, we must determine whether hESC-derived progeny truly resemble bona fide TB. Roberts et al. enumerate various accounts over the past decade that reported TB formation from hESCs, principally based on the expression of a few marker genes and cell morphology. Importantly, they have not addressed the more rigorous question of whether the chosen markers are sufficient to validate a TB identity, nor have they engaged in distinguishing between circumstantial and direct evidence for a bona fide placental TB phenotype. In the published reports, key markers of TB remained absent, genes from alternate lineages continued to be expressed and TB genes appeared inactive at the chromatin level (Bernardo et al. 2011). The current debate thus exemplifies the challenge of correctly identifying and validating the lineages that arise from hESC differentiation in vitro, owing to a shortage of the fundamental knowledge of human developmental biology that is needed as a benchmark to evaluate in vitro differentiation (McKnight et al. 2010, Mascetti \& Pedersen 2014). Taking into account this uncertainty, we advocated using more complex and function-based criteria (Bernardo et al. (2011) and in this debate) as a higher standard for TB identity than has been used in previous literature or by Roberts et al. (Amita et al. 2013).

Induction of TB-associated markers after differentiation of hESCs using BMP, together with TGF $\beta$ and FGF/MAPK antagonists (Erb et al. 2011, Sudheer et al. 2012, Amita et al. 2013), had been regarded as a pivotal advance in modeling TB development. TGF $\beta$ and FGF/MAPK inhibition likely suppresses primitive streak/ mesoderm induction (Bernardo et al. 2011, Loh et al. 2014), therefore minimizing the expression of chosen mesoderm genes (e.g. BRACHYURY and TBX4) and enhancing the expression of a few TB-associated markers (e.g. KRT7) (Amita et al. 2013). Rather than qualifying as a definitive marker of TB identity, however, $\mathrm{Krt7}$ is promiscuously expressed in diverse epithelial tissues (Ramaekers et al. 1987). Indeed, hESCs induced 
to express $\mathrm{Krt}$ by BMP treatment express not only TB-associated markers but also Is/1 and other mesodermal markers, possibly reflecting a mesodermal identity (Bernardo et al. 2011) or even a developmentally ambiguous mixed phenotype. The KRT7-expressing cell populations analyzed by Roberts et al. lacked expression of three particular mesoderm genes (TBX4, FLK1, and VCAM1; Amita et al. 2013), which signified to them that these cells do not belong to the mesoderm lineage. Their assertion relies on the assumption that presence or absence of a marker associated with a particular cell lineage is sufficient to define and validate cells as belonging to that lineage.

Expression of additional TB markers would perhaps lend confidence to an assignment of TB identity, provided that these were genes with known key functions in TB development, rather than genes expressed by a wide variety of cells and perhaps incidental to TB function. From this perspective, it is significant that transcription factors SOX2 and EOMES (key markers of mouse TSCs and essential for TB specification in vivo) are not detectably expressed during BMP-induced hESC differentiation (Sudheer et al. 2012, Amita et al. 2013). While Roberts et al. assert that these markers are expressed during the first $24 \mathrm{~h}$ of putative TB induction in vitro, both transcription factors are expressed in undifferentiated hESCs to some extent and their 'expression during the first day of differentiation' probably reflects residual expression from hESCs undergoing incipient differentiation. Indeed, both SOX2 and EOMES are acutely downregulated within $3 \mathrm{~h}$ of BMP4 + TGF $\beta$ inhibitor + FGFR/MAPK inhibitor treatment of hESCs and are progressively suppressed with prolonged treatment; at no stage is either gene upregulated (Sudheer et al. 2012). Additional key transcriptional regulators of TB (e.g. ELF5; $\mathrm{Ng}$ et al. 2008) are modestly upregulated (approximately tenfold) upon putative TB induction (Amita et al. 2013, Li et al. 2013), yet to the best of our knowledge, very low starting expression is typically observed for this gene in hESCs, and robust expression of ELF5 protein has not been reported. Finally, expression of CDX2, GATA3, and HAND1 has been cited as a proxy for TB commitment (Sudheer et al. 2012, Amita et al. 2013); however, these transcription factors are also expressed in posterior mesoderm (Bernardo et al. 2011), complicating their use as diagnostic markers. These ambiguities about the significance of expression of TB-associated marker genes (even multiple ones) by BMP-treated hESCs raises the questions of whether i) their TB identity is truly established and ii) the resultant cells share functional properties of bona fide TB. A partial TB identity or a mixture of extraembryonic identities would be reminiscent of the 'partial programming' that occurs when fetal progenitors are exposed to incomplete signaling regimens, leading to lineage infidelity whereby some but not all characteristic lineage markers are expressed (Wandzioch \& Zaret 2009).
Instead of addressing the specificity of cardinal TB markers and/or the functionality of putative hESC-derived TB, Roberts et al. have instead argued that their microarray profiles broadly cluster with placenta and they share placental gene ontology terms (Fig. 1C and D), therefore constituting evidence of their identity. However, these cells clearly lack expression of a cluster of signature genes expressed in first-trimester CTB ( $\mathrm{Li}$ et al. 2013) and there are marked transcriptional discrepancies between BMP-treated hESCs and either placenta or CTB (Fig. 1C). Furthermore, it was never assessed whether BMP-treated hESCs might cluster even closer to first-trimester amnion or early hESC-derived mesoderm or extraembryonic mesoderm populations therefore the relevant biological comparators are missing. To suggest that putative TB does not belong to a mesodermal lineage, Roberts et al. have cited RNA-seq analyses in which EOMES + BRACHYURY + anterior primitive streak/'mesendoderm' populations (Supplementary Fig. 1 of Xie et al. 2013) were shown to express a cohort of different genes than BMP-treated hESCs (Fig. 1A). However, in this study, any genes commonly expressed between the two cell populations were omitted from the analysis (Supplementary Methods of Xie et al. 2013), therefore obscuring any similarities between these lineages (Fig. 1A). Additionally, the relevant biological comparator is lacking again, because BMP-treated hESCs would not be expected to correspond to $\mathrm{EOMES}^{+}$anterior primitive streak but rather posterior mesoderm and extraembryonic mesoderm.

Faced with these uncertainties about the identity of BMP-induced progeny of hESCs, we have sought to define more complex criteria as an evidence for a bona fide TB phenotype (Bernardo et al. 2011).

i) In one approach, antibodies to a constellation of HLA epitopes were used to assess cell surface properties of BMP-induced hESC progeny, based on their presence in villous and/or extravillous human placental TB (Apps et al. 2009, Bernardo et al. 2011). Reportedly, BMP-induced hESC progeny stain positively for extravillous TB marker HLA-G (Amita et al. 2013). However, even when cells were differentiated on Matrigel and in KOSRcontaining medium as described by Amita et al. (2013), we were unable to detect HLA-G expression in quantitative flow cytometry assays (Bernardo et al. 2011). Moreover, the TB specificity of HLA-G immunostaining (Amita et al. 2013) remains uncertain. For example, amniotic epithelial cells also express HLA-G (Houlihan et al. 1995) and they (along with extraembryonic mesoderm lineages) similarly originate from a region of the mammalian embryo that is strongly influenced by BMP signaling (Lawson et al. 1991, Bosman et al. 2006). 
ii) Migratory behavior is another complex property that could potentially be informative of TB phenotype. BMP-induced hESCs display migratory properties in vitro (Amita et al. 2013, Telugu et al. 2013; thought to be evocative of invasive TB, Roberts et al., (this Debate)). Yet dissected primitive streak/nascent mesoderm similarly displays migratory capacities upon explant culture (Burdsal et al. 1993). This is likely a feature shared with all populations in the early embryo engaged in morphogenetic movements.

iii) The 'epigenetic' status of key genes involved in placental TB development is another 'complex' criterion for TB identity. In this regard, the promoter of ELF5 remains highly methylated in most (if not all) hESC-derived candidate TB (Hemberger et al. 2010, Bernardo et al. 2011), reflecting general transcriptional inactivity at both the mRNA and chromatin levels. This approach to assessing chromatin status could be readily extended to other key TB-associated genes using bisulfite sequencing and histone ChIP-seq methods. Even if additional TB-associated genes were shown to be unmethylated and expressed, however, such in vitro evidence for TB identity is purely circumstantial, as it does not demonstrate that the cells could function as bona fide TB during normal placental development.

Researchers have been faced with a similar quandary in delineating the fate of the embryo's own cells and of the stem cells derived from them. The developmental competence of the mouse embryo's native pluripotent cells has been studied extensively in classical studies using heterotopic grafting (Grobstein 1951, 1952) and in situ labeling (Lawson et al. 1991). In those studies, postimplantation embryonic epiblast never developed into TB derivatives. Likewise, late blastocyst stage embryonic epiblast was incapable of TB contribution after injection into recipient blastocysts (Gardner \& Rossant 1979), whereas ectoplacental cone and TSCs could contribute to TB in a similar assay (Rossant et al. 1978, Tanaka et al. 1998). In summary, both pre-implantation and postimplantation pluripotent cells in vivoare incapable of generating TB. Likewise, mESCs injected into blastocysts contributed to a very limited extent to the TB lineage (Beddington \& Robertson 1989). Nevertheless, despite their limited ability to contribute to the TB lineage in their native state, mESCs can be reprogramed to TB by overexpressing TB transcription factors (Niwa et al. 2005) or by relaxing epigenetic restrictions ( $\mathrm{Ng}$ et al. 2008). In recent studies of the developmental competence of EpiSCs grafted into post-implantation mouse embryos in vitro (Huang et al. 2012, Kojima et al. 2013), these integrated into the primitive streak but did not contribute to the TB lineage. The absence of evidence for TB functional identity is a further obstacle to conclude that post implantation-type PSCs (including mEpiSCs, hESCs, and hiPSCs) can be induced to differentiate in vitro into bona fide TB (Brons et al. 2007).

While it remains formally possible that a previously untested intervention (consisting of extrinsic signaling conditions with or without transcription factor or chromatin perturbations) could respecify hESCs to a phenotype that convincingly models bona fide placental $\mathrm{TB}$, this has not yet been demonstrated. Accordingly, the presently available data do not support the contention of Roberts et al. that it is 'unambiguously clear' that hESCs can give rise to TB. In our contributions to this debate, we emphasized the limitations of gene expression for validating in vitro differentiation and advocated using more complex, functional criteria to accurately identify TB. In our previously published work, we chose CDM, not for its efficiency of differentiation into cells with a putative TB phenotype, but because it was maximally defined, thereby enabling us to understand the signaling mechanisms responsible for particular cell fate decisions Bernardo et al. (2011). In their contribution, Roberts et al. have focused largely on reaffirming their choice of media (DMEM/F12/KOSR) and exogenous factors (the TGF $\beta$ inhibitor A-83-01 and the FGFR inhibitor PD173074) that enhance the efficiency of generating cells with a putative TB phenotype, based primarily on marker gene expression. Amita et al. (2013) have showed and Roberts et al. (current Debate) have reaffirmed that alterations in the conditions used for BMP-induced differentiation of hESCs can increase the incidence of $\mathrm{KRT7}^{+}$cells. A fraction (4-8\%) of KRT7 ${ }^{+}$ cells emerged in our CDM-based culture conditions, but these also expressed mesoderm-associated genes (Bernardo et al. 2011). In any case, increasing the frequency of $\mathrm{KRT}^{+}{ }^{+}$cells does not by itself address whether this represents bona fide placental TB.

If hESCs could truly give rise to TB, this would fundamentally revise our view of how developmental competence is relinquished during early embryonic lineage decisions. However, such a conclusion would require unequivocal proof that such cells met more complex in vitro criteria than simply expressing TB-associated markers and would require proof of their ability to function as placental TB. Roger Bacon once wrote 'argument is conclusive, but it does not remove doubt ... unless it finds it by the method of experiment' (http://en. wikiquote.org/wiki/Roger_Bacon) and likewise only objective evidence will enable us to advance from the present state of affairs and permit a more confident determination of TB identity among the progeny of hESCs. The aspiration for research funding to study this phenomenon is not a compelling argument for its existence.

\section{Note added in proof}

To further substantiate their notion that pluripotent stem cells harbor trophoblast potential, the Proposers cite two 
recent reports wherein iPSCs generated by in vivo reprogramming (Abad et al. 2013) or acid treatment (Obokata et al. 2014) were purported to be capable of trophoblast differentiation. The former report showed that upon in vitro differentiation or in vivo teratoma formation, iPSCs could give rise to $C d x 2$-expressing cells (Abad et al. 2013). However, $C d x 2$ does not qualify as an exclusive marker of trophoblast differentiation, for it is expressed in lineages from all three germ layers, as we have argued in this Debate and elsewhere (Bernardo et al. 2011; Loh et al. 2014). More convincingly, Abad et al. showed that after morula aggregation, iPSCs could grossly contribute to placenta. Crucially, however, Abad et al. did not co-stain for lineage-specific markers to ascertain whether donor cells contributed to the extra embryonic mesodermal component of the placenta (as expected for pluripotent cells) or whether they actually contributed to its trophoblast component. The report by Obokata et al. 2014 that mouse iPSCs could be generated using acid treatment remains to be verified (http://www.nature.com/news/acid-bath-stem-cellstudy-under-investigation-1.14738). Therefore it is difficult to interpret claims by Obokata et al. 2014 of the trophoblast potential of acid-induced iPSCs with confidence. Taken together, there is no definitive evidence from Abad et al. 2013 and Obokata et al. 2014 that iPSCs can indeed form trophoblast. We therefore conclude that available evidence does not meet the burden of proof needed to show that mammalian pluripotent cells in vivo or those cultivated in vitro are competent to undergo trophoblast differentiation in their native state. We cannot exclude the possibility, however, that true totipotent cells could be immortalized in vitro under other conditions and that those would have the potential to generate bona fide trophoblast.

\section{Declaration of interest}

The authors declare that there is no conflict of interest that could be perceived as prejudicing the impartiality of the research reported.

\section{Funding}

Proposers: Supported by NIH grant R01HD-067759 to R M Roberts and R01HD077108 to T Ezashi and D J Schust. Opposers: this research was supported by an MRC Programe grant, The Evelyn Trust, Cambridge, Hospitals National Institute for Health Research Biomedical Research Centre, and the Cambridge Centre for Trophoblast Research (R A Pedersen); and a British Heart Foundation Intermediate Basic Science Research Fellowship to A S Bernardo (grant number: FS/12/37/29516); and the Fannie and John Hertz Foundation, the U.S. National Science Foundation, and the Davidson Institute for Talent Development (K M Loh).

\section{Acknowledgements}

The proposers thank Mana Parast, University of California, San Diego, for helpful discussion throughout this 'Debate'. The opposers thank Ashley Moffett, Kathy Niakan, and Victoria Mascetti for their valuable comments.

\section{References}

Abad M, Mosteiro L, Pantoja C, Canamero M, Rayon T, Ors I, Grana O, Megias D, Dominguez O, Martinez D et al. 2013 Reprogramming in vivo produces teratomas and iPS cells with totipotency features. Nature 502 340-345. (doi:10.1038/nature12586)

Amit M, Carpenter MK, Inokuma MS, Chiu CP, Harris CP, Waknitz MA, Itskovitz-Eldor J \& Thomson JA 2000 Clonally derived human embryonic stem cell lines maintain pluripotency and proliferative potential for prolonged periods of culture. Developmental Biology 227 271-278. (doi:10.1006/dbio.2000.9912)

Amita M, Adachi K, Alexenko AP, Sinha S, Schust DJ, Schulz LC, Roberts RM \& Ezashi T 2013 Complete and unidirectional conversion of human embryonic stem cells to trophoblast by BMP4. PNAS 110 E1212-E1221. (doi:10.1073/pnas.1303094110)

Apps R, Murphy SP, Fernando R, Gardner L, Ahad T \& Moffett A 2009 Human leucocyte antigen (HLA) expression of primary trophoblast cells and placental cell lines, determined using single antigen beads to characterize allotype specificities of anti-HLA antibodies. Immunology 127 26-39. (doi:10.1111/j.1365-2567.2008.03019.x)

Avilion AA, Nicolis SK, Pevny LH, Perez L, Vivian N \& Lovell-Badge R 2003 Multipotent cell lineages in early mouse development depend on SOX2 function. Genes and Development 17 126-140. (doi:10.1101/gad. 224503)

Beddington RS \& Robertson EJ 1989 An assessment of the developmental potential of embryonic stem cells in the midgestation mouse embryo. Development 105 733-737.

Beppu H, Kawabata M, Hamamoto T, Chytil A, Minowa O, Noda T \& Miyazono K 2000 BMP type II receptor is required for gastrulation and early development of mouse embryos. Developmental Biology 221 249-258. (doi:10.1006/dbio.2000.9670)

Bernardo AS, Faial T, Gardner L, Niakan KK, Ortmann D, Senner CE, Callery EM, Trotter MW, Hemberger M, Smith JC et al. 2011 BRACHYURY and CDX2 mediate BMP-induced differentiation of human and mouse pluripotent stem cells into embryonic and extraembryonic lineages. Cell Stem Cell 9 144-155. (doi:10.1016/ j.stem.2011.06.015)

Besser D 2004 Expression of nodal, lefty-a, and lefty-B in undifferentiated human embryonic stem cells requires activation of Smad2/3. Journal of Biological Chemistry 279 45076-45084. (doi:10.1074/jbc.M4049 79200)

Bosman EA, Lawson KA, Debruyn J, Beek L, Francis A, Schoonjans L, Huylebroeck D \& Zwijsen A 2006 Smad5 determines murine amnion fate through the control of bone morphogenetic protein expression and signalling levels. Development 133 3399-3409. (doi:10.1242/dev. 02497)

Brons IG, Smithers LE, Trotter MW, Rugg-Gunn P, Sun B, Chuva de Sousa Lopes SM, Howlett SK, Clarkson A, Ahrlund-Richter L, Pedersen RA et al. 2007 Derivation of pluripotent epiblast stem cells from mammalian embryos. Nature 448 191-195. (doi:10.1038/nature05950)

Burdsal CA, Damsky CH \& Pedersen RA 1993 The role of E-cadherin and integrins in mesoderm differentiation and migration at the mammalian primitive streak. Development 118 829-844.

Carter AM 2007 Animal models of human placentation - a review. Placenta 28 (Suppl A) S41-S47. (doi:10.1016/j.placenta.2006.11.002)

Chen G, Ye Z, Yu X, Zou J, Mali P, Brodsky RA \& Cheng L 2008 Trophoblast differentiation defect in human embryonic stem cells lacking PIG-A and GPI-anchored cell-surface proteins. Cell Stem Cell 2 345-355. (doi:10.1016/j.stem.2008.02.004)

Das P, Ezashi T, Schulz LC, Westfall SD, Livingston KA \& Roberts RM 2007 Effects of fgf 2 and oxygen in the bmp4-driven differentiation of trophoblast from human embryonic stem cells. Stem Cell Research 1 61-74. (doi:10.1016/j.scr.2007.09.004) 
Di-Gregorio A, Sancho M, Stuckey DW, Crompton LA, Godwin J, Mishina Y \& Rodriguez TA 2007 BMP signalling inhibits premature neural differentiation in the mouse embryo. Development 134 3359-3369. (doi:10.1242/dev.005967)

Douglas GC, VandeVoort CA, Kumar P, Chang TC \& Golos TG 2009 Trophoblast stem cells: models for investigating trophectoderm differentiation and placental development. Endocrine Reviews 30 228-240. (doi:10.1210/er.2009-0001)

Drukker M, Tang C, Ardehali R, Rinkevich Y, Seita J, Lee AS, Mosley AR, Weissman IL \& Soen Y 2012 Isolation of primitive endoderm, mesoderm, vascular endothelial and trophoblast progenitors from human pluripotent stem cells. Nature Biotechnology 30 531-542. (doi:10.1038/nbt.2239)

Enders AC 2000 Trophoblast-uterine interactions in the first days of implantation: models for the study of implantation events in the human. Seminars in Reproductive Medicine 18 255-263. (doi:10.1055/s-200012563)

Erb TM, Schneider C, Mucko SE, Sanfilippo JS, Lowry NC, Desai MN, Mangoubi RS, Leuba SH \& Sammak PJ 2011 Paracrine and epigenetic control of trophectoderm Differentiation from human embryonic stem cells: the role of bone morphogenic protein 4 and histone deacetylases. Stem Cells and Development 20 1601-1614. (doi:10.1089/scd.2010. 0281)

Ezashi T, Telugu BP \& Roberts RM 2012 Model systems for studying trophoblast differentiation from human pluripotent stem cells. Cell and Tissue Research 349 809-824. (doi:10.1007/s00441-012-1371-2)

Gardner RL \& Rossant J 1979 Investigation of the fate of 4-5 day postcoitum mouse inner cell mass cells by blastocyst injection. Journal of Embryology and Experimental Morphology 52 141-152.

Genbacev O, Lamb JD, Prakobphol A, Donne M, McMaster MT \& Fisher SJ 2013 Human trophoblast progenitors: where do they reside? Seminars in Reproductive Medicine 31 56-61. (doi:10.1055/s-0032-1331798)

Georgiades P, Ferguson-Smith AC \& Burton GJ 2002 Comparative developmental anatomy of the murine and human definitive placentae. Placenta 23 3-19. (doi:10.1053/plac.2001.0738)

Gerami-Naini B, Dovzhenko OV, Durning M, Wegner FH, Thomson JA \& Golos TG 2004 Trophoblast differentiation in embryoid bodies derived from human embryonic stem cells. Endocrinology 145 1517-1524. (doi:10.1210/en.2003-1241)

Golos TG, Pollastrini LM \& Gerami-Naini B 2006 Human embryonic stem cells as a model for trophoblast differentiation. Seminars in Reproductive Medicine 24 314-321. (doi:10.1055/s-2006-952154)

Grobstein C 1951 Intra-ocular growth and differentiation of the mouse embryonic shield implanted directly and following in vitro cultivation. Journal of Experimental Zoology 116 501-525. (doi:10.1002/jez. 1401160308)

Grobstein C 1952 Intra-ocular growth and differentiation of clusters of mouse embryonic shields cultured with and without primitive endoderm and in the presence of possible inductors. Journal of Experimental Zoology 119 355-379. (doi:10.1002/jez.1401190303)

Gupta R, Ezashi T \& Roberts RM 2012 Squelching of ETS2 transactivation by POU5F1 silences the human chorionic gonadotropin CGA subunit gene in human choriocarcinoma and embryonic stem cells. Molecular Endocrinology 26 859-872. (doi:10.1210/me.2011-1146)

Hayashi Y, Furue MK, Tanaka S, Hirose M, Wakisaka N, Danno H, Ohnuma K, Oeda S, Aihara Y, Shiota K et al. 2010 BMP4 induction of trophoblast from mouse embryonic stem cells in defined culture conditions on laminin. In Vitro Cellular \& Developmental Biology. Animal 46 416-430. (doi:10.1007/s11626-009-9266-6)

Hemberger M, Udayashankar R, Tesar P, Moore H \& Burton GJ 2010 ELF5-enforced transcriptional networks define an epigenetically regulated trophoblast stem cell compartment in the human placenta. Human Molecular Genetics 19 2456-2467. (doi:10.1093/hmg/ddq128)

Houlihan JM, Biro PA, Harper HM, Jenkinson HJ \& Holmes CH 1995 The human amnion is a site of MHC class Ib expression: evidence for the expression of HLA-E and HLA-G. Journal of Immunology 154 5665-5674.

Huang Y, Osorno R, Tsakiridis A \& Wilson V 2012 In vivo differentiation potential of epiblast stem cells revealed by chimeric embryo formation. Cell Reports 2 1571-1578. (doi:10.1016/j.celrep.2012.10.022)

lacob D, Cai J, Tsonis M, Babwah A, Chakraborty C, Bhattacharjee RN \& Lala PK 2008 Decorin-mediated inhibition of proliferation and migration of the human trophoblast via different tyrosine kinase receptors. Endocrinology 149 6187-6197. (doi:10.1210/en.2008-0780)
Keramari M, Razavi J, Ingman KA, Patsch C, Edenhofer F, Ward CM \& Kimber SJ 2010 Sox2 is essential for formation of trophectoderm in the preimplantation embryo. PLoS ONE 5 e13952. (doi:10.1371/journal.pone. 0013952)

Kojima, Y, Kaufman-Francis K, Studdert JB, Steiner KA, Power MD, Loebel DA, Jones V, Hor A, de Alencastro G, Logan GJ et al. 2013 The transcriptional and functional properties of mouse epiblast stem cells resemble the anterior primitive streak. Cell Stem Cell 14 107-120. (doi:10.1016/j.stem.2013.09.014)

Lawson KA, Meneses JJ \& Pedersen RA 1991 Clonal analysis of epiblast fate during germ layer formation in the mouse embryo. Development $\mathbf{1 1 3}$ 891-911.

Li Y, Moretto-Zita M, Soncin F, Wakeland A, Wolfe L, Leon-Garcia S, Pandian R, Pizzo D, Cui L, Nazor K et al. 2013 BMP4-directed trophoblast differentiation of human embryonic stem cells is mediated through a DeltaNp63 + cytotrophoblast stem cell state. Development 140 3965-3976. (doi:10.1242/dev.092155)

Lichtner B, Knaus P, Lehrach H \& Adjaye J 2013 BMP10 as a potent inducer of trophoblast differentiation in human embryonic and induced pluripotent stem cells. Biomaterials 34 9789-9802. (doi:10.1016/ j.biomaterials.2013.08.084)

Loh KM, Ang LT, Zhang J, Kumar V, Ang J, Auyeong JQ, Lee KL, Choo SH, Lim CY, Nichane M et al. 2014 Efficient endoderm induction from human pluripotent stem cells by logically directing signals controlling lineage bifurcations. Cell Stem Cell 14 237-252.

Malassine A \& Cronier L 2002 Hormones and human trophoblast differentiation: a review. Endocrine 19 3-11. (doi:10.1385/ENDO:19:1:3)

Marchand M, Horcajadas JA, Esteban FJ, McElroy SL, Fisher SJ \& Giudice LC 2011 Transcriptomic signature of trophoblast differentiation in a human embryonic stem cell model. Biology of Reproduction $\mathbf{8 4}$ 1258-1271. (doi:10.1095/biolreprod.110.086413)

Mascetti VL \& Pedersen RA 2014 Naivete of the human pluripotent stem cell. Nature Biotechnology 32 68-70. (doi:10.1038/nbt.2789)

McKnight KD, Wang P \& Kim SK 2010 Deconstructing pancreas development to reconstruct human islets from pluripotent stem cells. Cell Stem Cell 6 300-308. (doi:10.1016/j.stem.2010.03.003)

Mishina Y, Suzuki A, Ueno N \& Behringer RR 1995 Bmpr encodes a type I bone morphogenetic protein receptor that is essential for gastrulation during mouse embryogenesis. Genes and Development 9 3027-3037. (doi:10.1101/gad.9.24.3027)

Ng RK, Dean W, Dawson C, Lucifero D, Madeja Z, Reik W \& Hemberger M 2008 Epigenetic restriction of embryonic cell lineage fate by methylation of Elf5. Nature Cell Biology 10 1280-1290. (doi:10. 1038/ncb1786)

Niakan KK, Han J, Pedersen RA, Simon C \& Pera RA 2012 Human pre-implantation embryo development. Development 139 829-841. (doi:10.1242/dev.060426)

Niwa H, Toyooka Y, Shimosato D, Strumpf D, Takahashi K, Yagi R \& Rossant J 2005 Interaction between Oct3/4 and Cdx2 determines trophectoderm differentiation. Cell 123 917-929. (doi:10.1016/j.cell. 2005.08.040)

Obokata H, Wakayama T, Sasai Y, Kojima K, Vacanti MP, Niwa H, Yamato M \& Vacanti CA 2014 Stimulus-triggered fate conversion of somatic cells into pluripotency. Nature 505 641-647. (doi:10.1038/nature12968)

Ramaekers F, Huysmans A, Schaart G, Moesker O \& Vooijs P 1987 Tissue distribution of keratin 7 as monitored by a monoclonal antibody. Experimental Cell Research 170 235-249. (doi:10.1016/0014-4827(87)90133-9)

Ringler GE \& Strauss JF III 1990 In vitro systems for the study of human placental endocrine function. Endocrine Reviews 11 105-123. (doi:10.1210/edrv-11-1-105)

Rossant J, Gardner RL \& Alexandre HL 1978 Investigation of the potency of cells from the postimplantation mouse embryo by blastocyst injection: a preliminary report. Journal of Embryology and Experimental Morphology 48 239-247.

Russ AP, Wattler S, Colledge WH, Aparicio SA, Carlton MB, Pearce JJ, Barton SC, Surani MA, Ryan K, Nehls MC et al. 2000 Eomesodermin is required for mouse trophoblast development and mesoderm formation. Nature 404 95-99. (doi:10.1038/35003601)

Schulz LC, Ezashi T, Das P, Westfall SD, Livingston KA \& Roberts RM 2008 Human embryonic stem cells as models for trophoblast differentiation. Placenta 29 (Suppl A) S10-S16. (doi:10.1016/j.placenta.2007.10.009) 
Silva J \& Smith A 2008 Capturing pluripotency. Cell 132 532-536. (doi:10.1016/j.cell.2008.02.006)

Sudheer S, Bhushan R, Fauler B, Lehrach H \& Adjaye J 2012 FGF inhibition directs BMP4-mediated differentiation of human embryonic stem cells to syncytiotrophoblast. Stem Cells and Development 21 2987-3000. (doi:10.1089/scd.2012.0099)

Tan T, Tang X, Zhang J, Niu Y, Chen H, Li B, Wei Q \& Ji W 2011 Generation of trophoblast stem cells from rabbit embryonic stem cells with BMP4. PLOS ONE 6 e17124. (doi:10.1371/journal.pone.0017124)

Tanaka S, Kunath T, Hadjantonakis AK, Nagy A \& Rossant J 1998 Promotion of trophoblast stem cell proliferation by FGF4. Science 282 2072-2075. (doi:10.1126/science.282.5396.2072)

Telugu V, Adachi K, Schlitt JM, Ezashi T, Schust DJ, Roberts RM \& Schulz LC 2013 Comparison of extravillous trophoblast cells derived from human embryonic stem cells and from first trimester human placentas. Placenta 34 536-543. (doi:10.1016/j.placenta.2013.03.016)

Vallier L, Touboul T, Chng Z, Brimpari M, Hannan N, Millan E, Smithers LE, Trotter M, Rugg-Gunn P, Weber A et al. 2009a Early cell fate decisions of human embryonic stem cells and mouse epiblast stem cells are controlled by the same signalling pathways. PLoS ONE 4 e6082. (doi:10.1371/journal.pone.0006082)

Vallier L, Mendjan S, Brown S, Chng Z, Teo A, Smithers LE, Trotter MW, Cho CH, Martinez A, Rugg-Gunn P et al. 2009b Activin/Nodal signalling maintains pluripotency by controlling Nanog expression. Development 136 1339-1349. (doi:10.1242/dev.033951)

Wandzioch E \& Zaret KS 2009 Dynamic signaling network for the specification of embryonic pancreas and liver progenitors. Science $\mathbf{3 2 4}$ 1707-1710. (doi:10.1126/science.1174497)
Wu Z, Zhang W, Chen G, Cheng L, Liao I, Jia N, Gao Y, Dai H, Yuan I \& Xiao L 2008 Combinatorial signals of activin/nodal and bone morphogenic protein regulate the early lineage segregation of human embryonic stem cells. Journal of Biological Chemistry 283 24991-25002. (doi:10.1074/jbc.M803893200)

Xie W, Schultz MD, Lister R, Hou Z, Rajagopal N, Ray P, Whitaker JW, Tian S, Hawkins RD, Leung D et al. 2013 Epigenomic analysis of multilineage differentiation of human embryonic stem cells. Cell 153 1134-1148. (doi:10.1016/j.cell.2013.04.022)

Xu RH 2006 In vitro induction of trophoblast from human embryonic stem cells. Methods in Molecular Medicine 121 189-202.

Xu RH, Chen X, Li DS, Li R, Addicks GC, Glennon C, Zwaka TP \& Thomson JA 2002 BMP4 initiates human embryonic stem cell differentiation to trophoblast. Nature Biotechnology 20 1261-1264. (doi:10.1038/nbt761)

Xu RH, Sampsell-Barron TL, Gu F, Root S, Peck RM, Pan G, Yu J, Antosiewicz-Bourget J, Tian S, Stewart R et al. 2008 NANOG is a direct target of TGF $\beta /$ activin-mediated SMAD signaling in human ESCs. Cell Stem Cell 3 196-206. (doi:10.1016/j.stem.2008.07.001)

Ying QL, Nichols J, Evans EP \& Smith AG 2002 Changing potency by spontaneous fusion. Nature 416 545-548. (doi:10.1038/nature729)

Yu P, Pan G, Yu J \& Thomson JA 2011 FGF2 sustains NANOG and switches the outcome of BMP4-induced human embryonic stem cell differentiation. Cell Stem Cell 8 326-334. (doi:10.1016/j.stem.2011.01.001)

Zhang P, Li J, Tan Z, Wang C, Liu T, Chen L, Yong J, Jiang W, Sun X, Du L et al. 2008 Short-term BMP-4 treatment initiates mesoderm induction in human embryonic stem cells. Blood 111 1933-1941. (doi:10.1182/ blood-2007-02-074120) 